\title{
REDigest: a Python GUI for In-Silico Restriction Digestion Analysis of Genes or Complete Genome Sequences
}

\author{
Abhijeet Singh ${ }^{1 *}$ \\ ${ }^{1}$ Anaerobic Microbiology and Biotechnology group, Department of Molecular Sciences, \\ Swedish University of Agricultural Sciences, Almas Allé 5, Uppsala, SE-750 07, Uppsala, \\ Sweden
}

*For correspondence. E-mail:abhijeet.singh@slu.se, abhijeetsingh.aau@gmail.com; Tel. +46 18671000; Fax +46 18672000; Address: Department of Molecular Sciences, Box 7025, 75007 Uppsala, Sweden

\begin{abstract}
Restriction fragment length polymorphism (RFLP) is a technology for the molecular characterization of DNA and widely used genome mapping, medical genetics, molecular microbiology and forensics etc. Terminal restriction fragment length polymorphism (T-RFLP), a variant of RFLP is extensively used in environmental microbiology for the microbial community profiling based on the restriction digestion profile of marker gene (16S rRNA, FTHFS etc.) amplicons. At present, there is a lack of a tool which can perform in-silico restriction digestion of a large number of sequences at a time, in an interactive way and as an output produce sequences of the restriction fragments and visualization plot. I have developed a graphical user interface based software "REDigest" for the in-silico restriction digestion analysis for gene or genome sequences. The REDigest software program with a graphical user interface is freely available at https://github.com/abhijeetsingh1704/REDigest.
\end{abstract}




\section{INTRODUCTION}

Restriction fragment length polymorphism (RFLP) is a molecular technique used to identify and visualize the variation in DNA sequences. This technique exploits the restriction enzyme target sites in DNA sequences to produce fragments of different length which can be visualized using agarose gel electrophoresis. It is a fast and relatively inexpensive method for the screening and comparing a large number of samples based on their restriction profiles. RFLP or similar techniques have been used in different applications, such as marker-assisted selection (MAS) in animal breeding (Soller, 1978; Beuzen et al., 2000), human genetics (Botstein et al., 1980), parenthood testing (Jeffreys et al., 1985), MAS in plant breeding (Bernatzky and Tanksley, 1986). During the years several variations of this techniques have been developed i.e. Amplified Fragment Length Polymorphism (AFLP) (Vos et al., 1995) and Terminal restriction fragment length polymorphism (T-RFLP) (Liu et al., 1997). Especially T-RFLP is being used for the microbial community profiling in environmental and medical microbiology (Osborn et al., 2000; Ricke et al., 2005; Zhang et al., 2008). There are a few programs available for the insilico analysis of restriction fragments like TRiFLe (Junier et al., 2008), in-silico T-RFLP (Bikandi et al., 2004) and NEBcutter (Vincze et al., 2003) etc. However, they are limited in terms of output in the form of just numerical values of the terminal restriction fragment size or are low-throughput and can process only a single sequence at a time. During this in-silico digestion, only the restriction site position or a restriction map is generated as an output and not sequence information (in any form) is generated. Therefore, there is a need for a program which can process large sequence datasets and generate restriction profile of the whole dataset and still preserves the sequence information in output. Here, I present REDigest, a graphical user interface (GUI) based software program for the in-silico restriction digestion of a large number of gene sequences or complete genomes. The name of this software program "REDigest", is derived from Restriction Enzyme Digestion. 
REDigest takes sequence data of genes or a genome in a Fasta (Wikipedia, 2020b; Lipman and Pearson, 1985) or Genbank file format (NCBI, 2006) and produces 1) a Fasta/Genbank format file of the terminal restriction fragment, 2) .csv format file/files (Wikipedia, 2020a) of the restriction and terminal restriction fragments and 3) a restriction fragment diversity plot. REDigest is developed for and can be used on Windows and Linux Operating systems, and tested on Windows 10 (Microsoft, 2015) and Ubuntu Linux derivative Peppermint (Peppermint LLC, 2010). REDigest is licensed under MIT license and can be freely available to download and use from https://github.com/abhijeetsingh1704/REDigest.

\section{IMPLEMENTATION}

REDigest is written in Python3 (The PSF, 2020) (version 3.8.5) and uses module Biopython (Cock et al., 2009) (version 1.77) (Chang et al., 2020) for the restriction digestion and sequence manipulation, Pandas (The PDT, 2020) (version 1.1.1) for numerical data analysis and Matplotlib (Hunter, 2007) (version 3.3.1) for visualization. The graphical user interface is developed using the Python module Tkinter (Tcl/Tk, 2019) (version 8.6). To use REDigest, it is a prerequisite that all these dependencies must be installed and are present in the execution path.

\section{USER INTERFACE AND FUNCTIONALITY}

\section{Restriction Digestion and Visualization}

REDigest has a very simple and interactive user interface and allow restriction digestion plus visualization in a single program execution from Restriction \& Visualization tab. REDigest can process Fasta and Genbank format files as input and can write output file for sequence information in Fasta or Genbank format. The restriction analysis can be performed on a set of multiple genes or a single genome. In case the input file is a multifasta gene file, there is an 
option to indicate the labelled primer. If the forward primer is labelled, the output will be the sense strand of the terminal restriction fragment and if the reverse primer is chosen as the labelled primer, the output will be the reverse complement sequence of the terminal restriction fragment in 5' to 3' orientation. Primer sequences for the forward and reverse primers can also be given which will be added and processed accordingly in the in-silico restriction digestion analysis. These options are optional for multigene sequence file. However, these options can be avoided in the case of processing the genome sequence.

The output generated from the REDigest analysis will be different based on the input files. If the input is the multifasta gene file, the output will be 1) a sequence file containing the terminal restriction fragment sequences with unique accession numbers, 2) a scatter plot representing the diversity and abundance of terminal restriction fragments and 3) a data-table containing the information of terminal restriction fragments. This data-table contains the information of the length of terminal restriction fragment and position of consecutive restriction enzyme cutting site. If a whole genome sequence is processed, the output from REDigest will be 1) a sequence file containing the sequences of an individual restriction fragment, 2) a scatter plot representing the diversity and abundance of restriction fragments and 3) two data-tables containing the length of individual restriction fragments and length of terminal restriction fragments of the genome. The interactive GUI of REDigest is presented in figure 1A. Users are required to provide the path to the input file and the case-sensitive name of the restriction enzyme, which are mandatory parameters to start the process, indicated in red colour. Rest of the parameters are optional, and the user needs to click the respective button for it to be activated. If the parameters are not provided or if they are not activated, default values will be used during the processing of the input file. The information about all the default values can be accessed from the help menu for respective tabs. Activation of the option will change the label colour to green and the click button will be changed to Ok (figure 1B). On the successful execution of the in- 
bioRxiv preprint doi: https://doi org/10.1101/2021.11.09.467873; this version posted November $11,2021$. The copyright holder for this preprint (which was not certified by peer review) is the author/funder, who has granted bioRxiv a license to display the preprint in perpetuity. It is made available under aCC-BY-NC-ND 4.0 International license.

silico restriction digestion and visualization process, a pop-up message box will appear on the screen (figure 2B).

A)

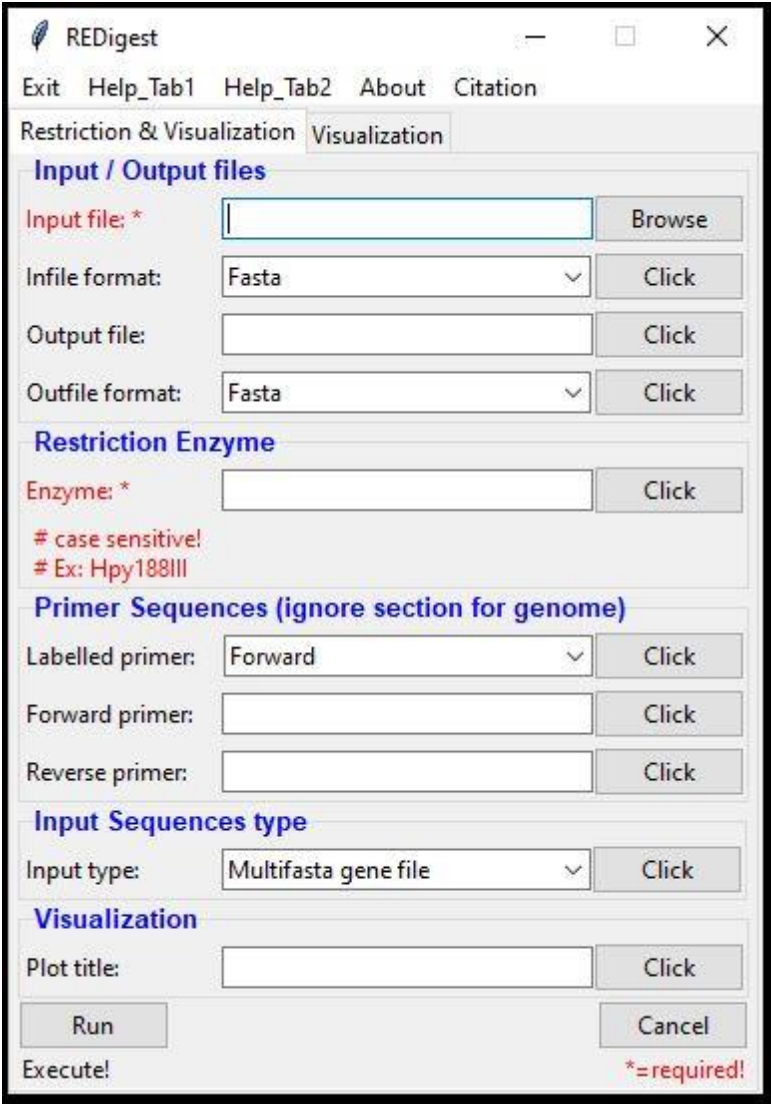

B)

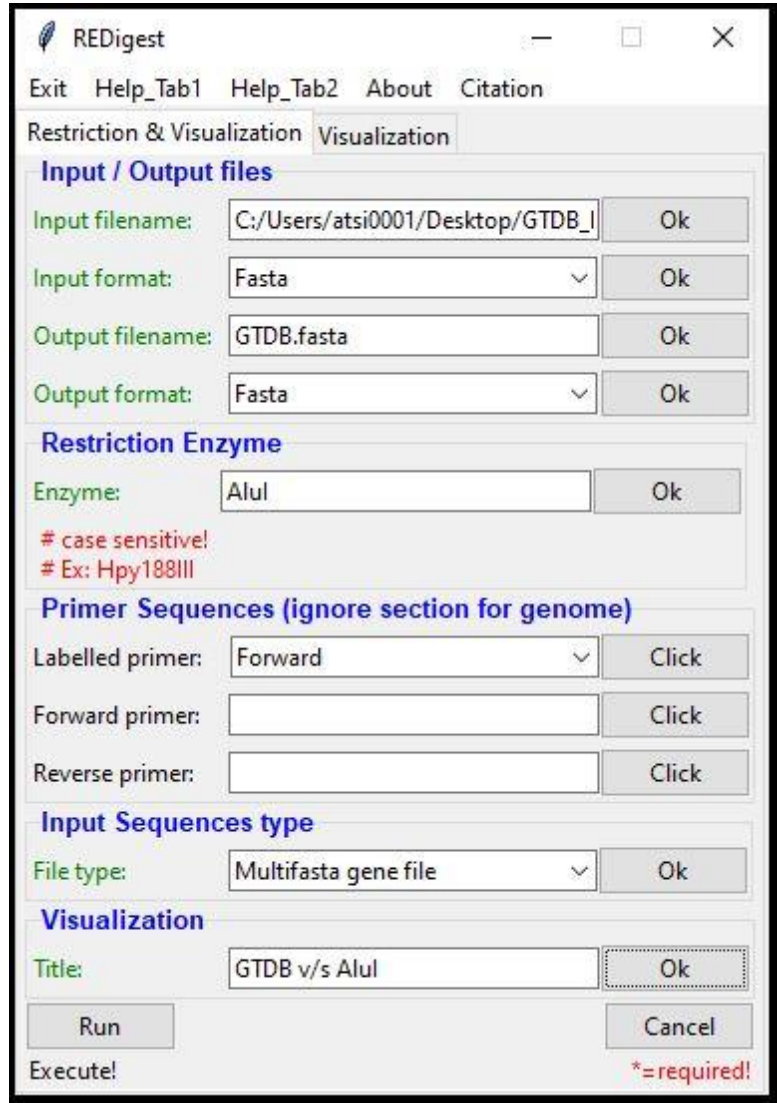

Figure 1: Screen-shot images representing the interactive graphical user interface (GUI) of REDigest software program on the Windows 10 operating system. A) the interface upon calling/starting the software GUI. Black colour input labels indicate the value is empty and need to be provided, word required in red colour indicates the respective mandatory field. If the labels (except input file and Enzyme name) are not activated, default values will be used. B) User-specific parameters for the respective parameters and its activation is indicated by the change in the colour of labels from black to green. The activation of the parameters is done by the click button which will change to Ok button upon activation.

\section{Custom Visualization}

User can also perform custom visualization from the Visualization tab for the data-table files generated by REDigest from Restriction \& Visualization tab. In this visualization process, it is a prerequisite that the data structure in the .csv files is not changed. For the custom visualization, all the plot attributes can be customized as shown in figure $\mathbf{2 A}$. After the successful generation 
bioRxiv preprint doi: https:/doi org/10.1101/2021.11.09.467873; this version posted November $11,2021$. The copyright holder for this preprint (which was not certified by peer review) is the author/funder, who has granted bioRxiv a license to display the preprint in perpetuity. It is made available under aCC-BY-NC-ND 4.0 International license.

of the custom plot, a pop-up message will indicate the completion of the plotting process figure

2B.

A)

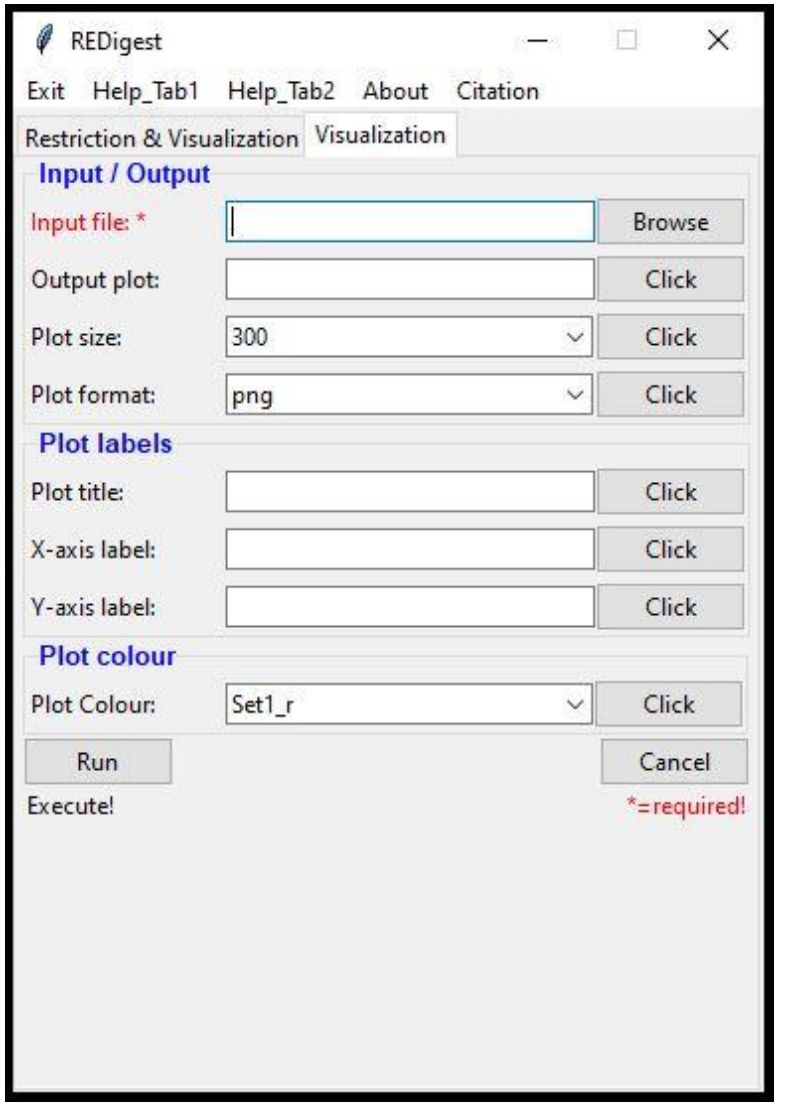

B)

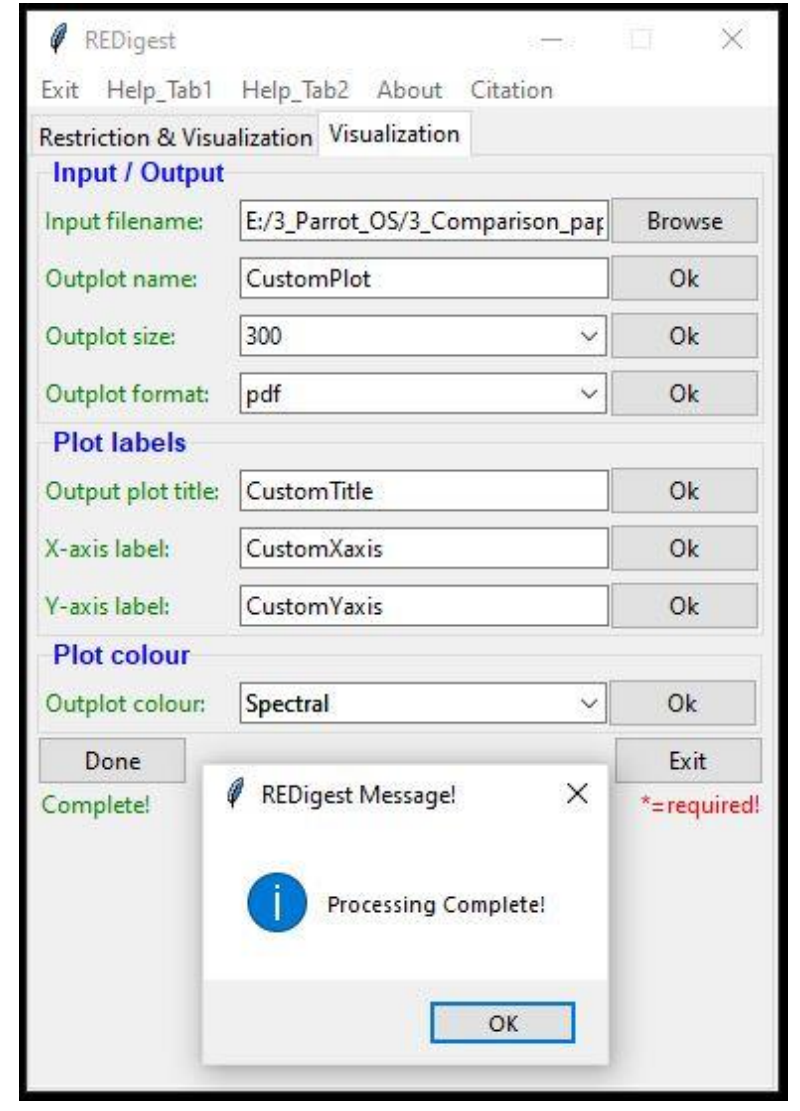

Figure 2: Screen-shot images representing the interactive graphical user interface (GUI) of REDigest software program for the Visualization tab on the Windows 10 operating system. A) the window for the Visualization tab where user can provide the custom plot attributes. The input file must be provided by the user, or an error message will appear on the screen. If the respective parameters are empty or not activated, default values will be used. B) Custom parameters provided by the user and its activation will be indicated by the respective change of text, colour and click button. A pop-up message will appear on the screen upon successful execution of the process.

\section{USAGE EXAMPLES}

REDigest have been tested against two data-sets of 16S rRNA gene sequences and FTHFS gene sequences from Silva-database training data formatted for DADA2 (McLaren, 2020) and AcetoBase (Singh et al., 2019), respectively. 16S rRNA gene training dataset (against restriction enzyme AluI) and AcetoBase dataset contain 374222 and 6806 sequences, 
respectively. These two datasets (Silva and AcetoBase) were processed in REDigest without any marker primer sequence and without any addition of primer sequences with restriction enzyme AluI and Hpy188III, respectively. For the genome analysis, the genome of Pelotomaculum thermopropionicum SI (NC_009454.1) was retrieved from NCBI RefSeq assembly and processed against the restriction enzyme AluI. All the analyses were performed on a Windows 10 operating system with $3.4 \mathrm{GHz}$ Inter ${ }^{\circledR}$ Core $^{\mathrm{TM}}$ i7-6700 processor and $29 \mathrm{~Gb}$ of available RAM. For these two datasets, the output files generated were the same as described earlier for the multigene fasta file. The plot generated from the respective analysis is presented in figure 3 .

A)

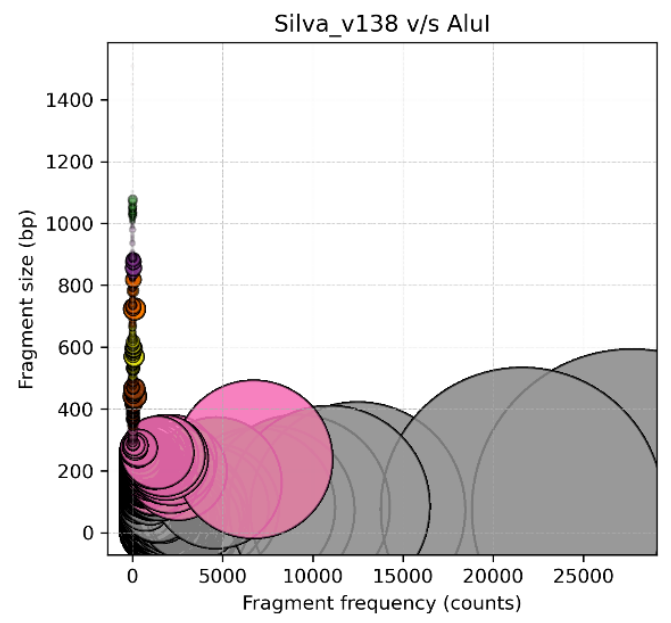

C)

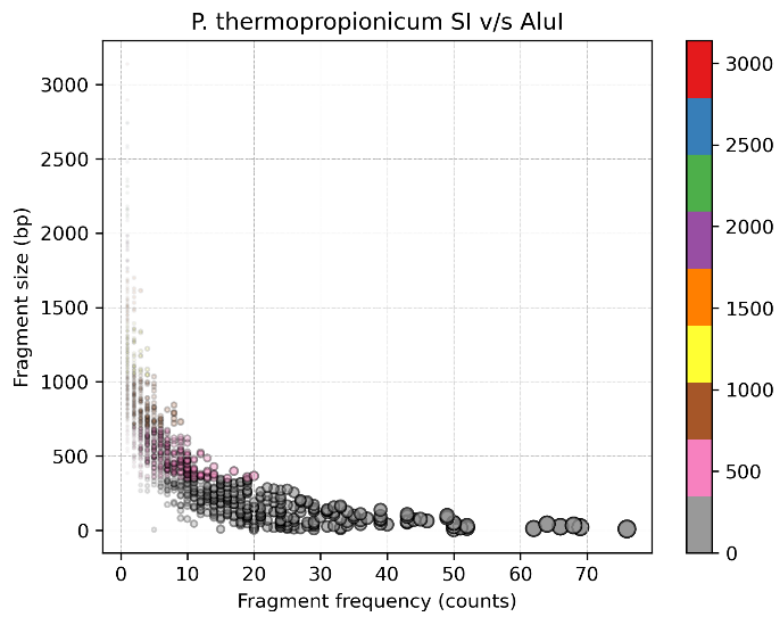

B)
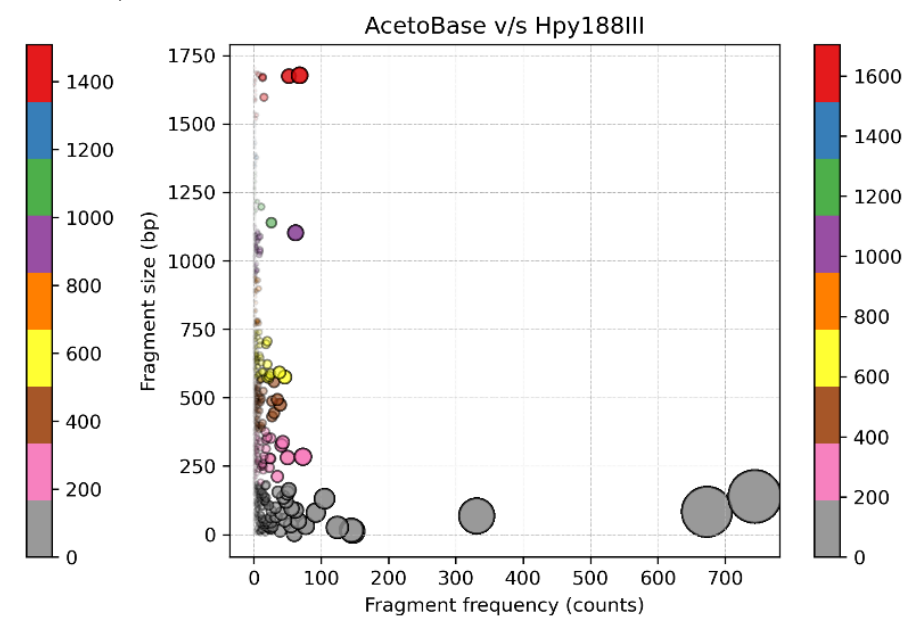

Figure 3: The scatter plot generated from REDigest for A) Silva database training dataset processed for the restriction enzyme AluI. B) AcetoBase nucleotide dataset processed for the 
restriction enzyme Hpy188III. C) Pelotomaculum thermopropionicum SI complete genome sequence (NC_009454.1) processed against the restriction enzyme AluI.

\section{CONCLUSION}

REDigest is a fast, user-interactive and customizable software program which can perform insilico restriction digestion analysis on a multifasta gene or a complete genome sequence file. REDigest can be helpful in different ways, for example:

1) It can help in the selection of suitable restriction enzyme for the restriction digestion experiment, by processing respective reference gene/genome data with several restriction enzymes and evaluate and choose the best restriction enzyme.

2) Validation of the restriction fragment or the terminal restriction fragment size and taxonomy against a database.

3) A restriction fragment or a terminal restriction database can be generated by using REDigest which can be helpful in the comparison and validation of the experimental restriction digestion profiles

\section{ACKNOWLEDGEMENTS}

I would like to thank Prof. Anna Schnürer for her support and motivation in the development of REDigest and valuable comments on the manuscript.

\section{COMPETING INTEREST STATEMENT}

None to be declared 


\section{BIBLIOGRAPHY}

Bernatzky R and Tanksley SD (1986) Toward a saturated linkage map in tomato based on isozymes and random cDNA sequences. Genetics 112 (4), 887-898.

Beuzen ND, Stear MJ, and Chang KC (2000) Molecular markers and their use in animal breeding. Vet. J. 160 (1), 42-52. https://doi.org/10.1053/tvj1.2000.0468

Bikandi J, Millán RS, Rementeria A, and Garaizar J (2004) In silico analysis of complete bacterial genomes: PCR, AFLP-PCR and endonuclease restriction. Bioinformatics 20 (5), 798-799. https://doi.org/10.1093/bioinformatics/btg491

Botstein D, White RL, Skolnick M, and Davis RW (1980) Construction of a Genetic Linkage Map in Man Using Restriction Fragment Length Polymorphisms. Am J Hum Gen 32, 314331.

Chang J, Chapman B, Friedberg I, Hamelryck T, Hoon M de, et al. (2020) Biopython 1.77. Biopython.org, version 1.77, 25 May 2020, https://biopython.org/wiki/Documentation.

Cock PJA, Antao T, Chang JT, Chapman BA, Cox CJ, et al. (2009) Biopython: Freely available Python tools for computational molecular biology and bioinformatics. Bioinformatics https://doi.org/10.1093/bioinformatics/btp163

Hunter JD (2007) Matplotlib: A 2D Graphics Environment. Comput. Sci. Eng. 9 (3), 90-95. https://doi.org/10.1109/MCSE.2007.55

Jeffreys AJ, Wilson V, and Thein SL (1985) Individual-specific 'fingerprints' of human DNA. Nature https://doi.org/10.1038/316076a0

Junier P, Junier T, and Witzel KP (2008) TRiFLe, a program for in silico terminal restriction fragment length polymorphism analysis with user-defined sequence sets. Appl. Environ. Microbiol. 74 (20), 6452-6456. https://doi.org/10.1128/AEM.01394-08

Lipman DJ and Pearson WR (1985) Rapid and sensitive protein similarity searches. Science. https://doi.org/10.1126/science.2983426

Liu WT, Marsh TL, Cheng H, and Forney LJ (1997) Characterization of microbial diversity by determining terminal restriction fragment length polymorphisms of genes encoding $16 \mathrm{~S}$ rRNA. Appl. Environ. Microbiol. $\mathbf{6 3} \quad$ (11), 4516-4522. https://doi.org/10.1128/aem.63.11.4516-4522.1997

McLaren MR (2020) Silva SSU taxonomic training data formatted for DADA2 Silva version 138, https://zenodo.org/, DOI: 10.5281/zenodo.3731176.

Microsoft (2015) Windows 10. Wikipedia, 25 August 2020, at 11:28 (UTC), https://en.wikipedia.org/wiki/Windows_10.

NCBI (2006) GenBank Flat File Format National Center for Biotechnology Information, Revised October 23, 2006, https://www.ncbi.nlm.nih.gov/Sitemap/samplerecord.html.

Osborn AM, Moore ERB, and Timmis KN (2000) An evaluation of terminal-restriction fragment length polymorphism (T-RFLP) analysis for the study of microbial community structure and dynamics. Environ. Microbiol. 2 (1), 39-50. https://doi.org/10.1046/j.14622920.2000.00081.x 
Peppermint LLC (2010) Peppermint OS, v10. Wikipedia, 19 June 2020, at 08:48 (UTC), https://en.wikipedia.org/wiki/Peppermint_OS.

Ricke P, Kolb S, and Braker G (2005) Application of a newly developed ARB softwareintegrated tool for in silico terminal restriction fragment length polymorphism analysis reveals the dominance of a novel pmoA cluster in a forest soil. Appl. Environ. Microbiol. 71 (3), 1671-1673. https://doi.org/10.1128/AEM.71.3.1671-1673.2005

Singh A, Müller B, Fuxelius HH, and Schnürer A (2019) AcetoBase: a functional gene repository and database for formyltetrahydrofolate synthetase sequences. Database (Oxford). https://doi.org/10.1093/database/baz142

Soller M (1978) The use of loci associated with quantitative effects in dairy cattle improvement. Anim. Prod. 27 (2), 133-139. https://doi.org/10.1017/S0003356100035960

Tcl/Tk (2019) Tkinter. The Tcl Developer Xchange, version 8.6, Nov 21, 2019, https://www.tcl.tk/software/tcltk/8.6.html.

The PDT (2020) Pandas. Zenodo/The Pandas Development Team (PDT), https://doi.org/10.5281/zenodo.3509134.

The PSF (2020) Python 3.8.5. The Python Software Foundation (PSF), Aug 26, 2020, https://docs.python.org/3/.

Vincze T, Posfai J, and Roberts RJ (2003) NEBcutter: A program to cleave DNA with restriction enzymes. Nucleic Acids Res. https://doi.org/10.1093/nar/gkg526

Vos P, Hogers R, Bleeker M, Reijans M, van de Lee T, et al. (1995) AFLP: a new technique for DNA fingerprinting. Nucleic Acids Res. 23 (21), 4407-4414. https://doi.org/10.1093/nar/23.21.4407

Wikipedia (2020a) Comma-separated values (.csv) Wikipedia, 31 May 2020, at 01:27 (UTC), https://en.wikipedia.org/wiki/Comma-separated_values.

Wikipedia (2020b) FASTA file format Wikipedia, 16 August 2020, at 07:22 (UTC), https://en.wikipedia.org/wiki/FASTA_format.

Zhang R, Thiyagarajan V, and Qian PY (2008) Evaluation of terminal-restriction fragment length polymorphism analysis in contrasting marine environments. FEMS Microbiol. Ecol. 65 (1), 169-178. https://doi.org/10.1111/j.1574-6941.2008.00493.x 